\title{
DER KODEX ÜBER AUSLANDSINVESTITIONEN DER SOZIALISTISCHEN REPUBLIK VIETNAM
}

\author{
Von BRun-Otto BRYde
}

1. Der nachstehende vietnamesische Investitionskodex wird nicht als Beispiel eines typischen Investitionsförderungsgesetzes eines um ausländische Investitionen bemühten Entwicklungslandes abgedruckt, obwohl er sich dafür durchaus eignen würde. Er enthält nämlich die für derartige Normen üblichen Regelungen ${ }^{1}$ : Eigentums- (bzw. Entschädigungs-)garantie (Art. $10 \mathrm{Z} .1$ \& 2), Steuervergünstigungen (Art. 11, 15), erleichterter Gewinn- und Kapitaltransfer (Art. 10 Z. 3 \& 4), Schiedsklausel (Art. 24, 25). Aber gerade das Typische ist in diesem Fall außergewöhnlich, denn daß das sozialistische Vietnam sich auf den internationalen Wettbewerb um die Gunst multinationaler Investoren (incentive scramble) einläßt, ist immerhin nicht selbstverständlich. Diese Bereitschaft dürfte über die im Kodex niedergelegten Angebote noch hinausgehen, denn alle Informationen deuten darauf hin, daß vietnamesische Unterhändler in ihrem Bemühen um Auslandskapital von der in Art. 26 gegebenen Möglichkeit, dem ausländischen Investor günstigere Bedingungen einzuräumen, großzügigen Gebrauch machen². Das soll nicht heißen, daß der Kodex keinen Hinweis auf die sozialistische Natur der vietnamesischen Wirtschaftsordnung enthielte. Ausländische Betätigung ist auf bestimmte Typen beschränkt. Kooperation, nämlich joint-ventures, die eine vietnamesische Kapitalmehrheit haben müssen (Art. 5, 7), und production-sharing agreements (Art. 5, 6), die vor allem für die Erdölgewinnung gedacht sind, stehen im Vordergrund. Rein private Unternehmen dürfen nur für den Export tätig werden (Art. 5, 8) wobei Hauptinvestitionsanreiz das vom Staat garantierte niedrige Lohnniveau bei qualifizierten Arbeitskräften ist (Art. 12 Z. 1). Nicht-Enteignung wird i. d. R. nur für 10-15 Jahre garantiert (Art. 10 Z. 1), doch werden längere Garantiezeiträume im Werben um Auslandskapital angeboten ${ }^{3}$. Die Bestimmung dürfte daher vor allem dogmenerhaltende Funktion haben, da Vietnam die Perspektive einer vollständig vergesellschafteten Wirtschaft nicht formell aufgeben möchte. Ob diese zeitliche Begrenzung ein so starkes psychologisches Investitionshindernis sein wird, wie gelegentlich angenommen ${ }^{4}$, ist fraglich, denn für den kühl kalkulierenden westlichen Geschäftsmann dürfte eine 15-Jahres-Garantie der vietnamesischen Regierung wertvoller sein, als eine Ewigkeitsgarantie von Kaiser Bokassa. Auch im übrigen enthält der Kodex Bestimmungen, die die ausländischen Investoren der nationalen Wirtschaftspolitik ein- und unterordnen sollen, aber die entsprechenden Bestimmungen (Art. 14-16) gehen nicht über das hinaus, was generell in Investitionsgesetzen, auch solchen „kapitalistischer“ Entwicklungsländer, üblich ist ${ }^{5}$ und was auch in verwirklichten oder geplanten internationalen Kodices für das Verhalten multinationaler Konzerne verlangt wird, nämlich die Beachtung der Wirtschaftspolitik und der Rechtsordnung des Gastlandes ${ }^{6}$.

1 Vgl. dazu den Uberblick bei Frick, Bilateraler Investitionsschutz in Entwicklungsländern, Berlin 1975, S. $36 \mathrm{ff}$. m. w. N., und H. Weber, in diesem Heft, S. $13 \mathrm{f}$.

2 Far Eastern Economic Review v. 29. 7. 77, S. 34 f.

3 Far Eastern Economic Review a. a. O.

4 International Herald Tribune v. 23. 3. 1977; Far Eastern Economic Review a. a. O.

5 Frick, a. a. O., S. $40 \mathrm{f}$. 6 Vgl. Leitsätze 1-9 der OECD Grundsätze für Multinationale Unternehmen v. 21. 6. 1976; zu den Plänen für einen UN-Codex vgl. den Vorbericht der Commission on Transnational Corporations des Wirtschafts- und Sozialrats, E/C. 10/17 v. 20. 7. 1976, insbes. Z. 67-78. 
Wenn der Kodex daher auch sicher keinen Übergang Vietnams zum Kapitalismus ankündigt (so daß die „Multis" siegen würden, wo die „Marines“ unterlegen sind), so zeigt er doch, daß Vietnam keinen Widerspruch zwischen einem sozialistischen Entwicklungsweg und forcierter wirtschaftlicher Kooperation mit westlichen Ländern und weit geöffneten Toren für privates Auslandskapital sieht. Insbesondere für die Ausbeutung seiner Rohölreserven und für die Ausnützung seines Niedriglohnstatus für.devisenbringende Exporte ${ }^{7}$ baut Vietnam offensichtlich auf die $\mathrm{Zu}$ sammenarbeit mit multinationalen Unternehmen. Dieser Sachverhalt verdient eine außenpolitische Randglosse und ein entwicklungstheoretisches Ausrufezeichen.

2. Außenpolitisch paßt das Werben um westliches Kapital in eine Reihe von Signalen Vietnams, die zeigen, daß es an einer neutralen Haltung und Offenheit gegenüber dem Westen interessiert ist. Hierher gehören die Integration in die Institution der westlichen Weltwirtschaftsordnung (Weltbank, IWF, Asian Development Bank), ebenso pragmatisch wie elegant dadurch bewerkstelligt, daß das wiedervereinigte Vietnam den Sitz Süd-Vietnams einnahm ${ }^{8}$, die Normalisierung der Beziehungen zu den ASEAN-Ländern', die Intensivierung der Beziehungen zur ehemaligen französischen Kolonialmacht ${ }^{10}$. Offensichtlich sieht die vietnamesische Führung, für die "nationale Unabhängigkeit" absoluten Vorrang vor allen anderen politischen Zielen haben dürfte ${ }^{11}$, diese Unabhängigkeit nach gewonnenem Vietnamkrieg eher durch die großen Brüder und Verbündeten von gestern, die UdSSR und China, bedroht als vom Westen, der sich als Papiertiger erwiesen hat. Dabei scheinen sich insbesondere die Beziehungen zu China, mit dem es ungelöste Grenzprobleme gibt, und das im Grenzkrieg zwischen Vietnam und Kampuchea ziemlich deutlich für letzteres Partei ergreift, rapide zu verschlechtern. Vietnam als Jugoslawien Süd-Ostasiens ${ }^{12}$ (nur stärker und noch selbstbewußter), das zeigt noch einmal nachträglich das tragische Ausmaß der Fehlkalkulation der USA, die in die Katastrophe des Vietnamkrieges unter dem Programm eines „containment" der kommunistischen Großmächte hineinschlitterten.

3. Wichtiger ist die Bedeutung der vietnamesischen Haltung für die aktuelle Diskussion um Entwicklungsstrategien. Wenn ein Land wie Vietnam seine Entwicklungshoffnungen auf Weltmarktintegration, insbes. Exportförderung und Devisenbeschaffung setzt, dann ist die praktische Relevanz von Entwicklungstheorien, die den Entwicklungsländern „Dissoziation“ empfehlen ${ }^{13}$ immerhin zweifelhaft. Nun kann die "Richtigkeit" einer Entwicklungstheorie sicher nicht einfach durch das Verhalten von Regierungen der Dritten Welt widerlegt werden. Aber es dürfte Konsens darüber bestehen, daß Entwicklungspolitik eine praktische Wissenschaft ist, und daß daher Modelle, die nur in der Luft reiner Theorie gedeihen, in der realen Welt aber keinerlei Verwirklichungschancen haben, keine allzu sinnvollen

7 Nach Wohlmuth, Multinationale Konzerne, Nationaler Markt und Unterentwicklung, in Senghaas/ Menzel (Hrsg.). Multinationale Konzerne und Dritte Welt, Opladen 1976, S. 67 ff., 92 ff., die schädlichste aller Formen multinationaler Aktivitäten.

8 Während Vietnam in COMECON nur Beobachterstatus hat.

9 Far Eastern Economic Review v. 20. 1. 1978, S. 10.

$10 \mathrm{NZZ}$ v. 1. 5. 1977.

11 Ho Tschi Minh fiel schon in den 20er Jahren in Moskau durch seine betont nationalistische Haltung irritierend auf, vgl. R. Fischer, Von Lenin zu Mao, Düsseldorf 1956, S. 176.

12 Die NZZ spricht von der ${ }^{n}$ Titosierung ${ }^{\alpha}$ Indochinas (1. 5. 1977). und Entwicklungspolitik. Plädoyer für Dissoziation, Frankfurt 1977, S. $262 \mathrm{ff}$. 
Beiträge sind. Daß die Regierungen der Dritten Welt generell verbesserte und chancengleichere Weltmarktintegration anstreben und nicht „Dissoziation “14 und daß ein gewisses Paradox darin liegt, ihnen „autozentrierte“ Entwicklung zu empfehlen, aber gleichzeitig nicht von ihren eigenen Zielen auszugehen, sondern von außen andere Ziele vorzuschreiben, steht schon länger fest. Der plausible Gegeneinwand war, daß solche Regierungen nicht die wirklichen Interessen ihrer Länder verfolgen, sondern als Verbündete der multinationalen Konzerne ihre eigenen Interessen zum Schaden der Masse der Bevölkerung ${ }^{15}$. Hier ist nun das vietnamesische Beispiel von Bedeutung; zwar nicht, weil man bei einer kommunistischen Regierung ex definitione davon ausgehen könnte, daß sie nicht ihre eigenen I teressen, sondern die der Masse der Bevölkerung im Auge hätte. Die Erfahrungen mit der Bürokratenklasse der osteuropäischen Länder schließen solchen, ironischerweise gerade bei materialistisch orientierten Autoren verbreiteten Idealismus aus. Aber man kann immerhin sicher sein, daß die vietnamesischen Führer sich nicht nach 30jährigem opferreichen Kampf als heimliche „Lakaien des Imperialismus und Monopolkapitalismus“ entpuppen. Unabhängig von der abstrakten „Richtigkeit“ eines dissoziativen Modells scheint jedenfalls seine praktische Relevanz gering zu sein, wenn das vietnamesische Beispiel den Schluß nahelegt, daß nicht einmal eine Ersetzung der jetzigen politischen Systeme der Dritten Welt durch eine sozialistische Revolution Akteure schaffen würde, die sich einem derartigen Modell verschreiben. Die Überzeugungskraft der verbleibenden Beispiele für eine dissoziative Entwicklung ist gering, denn den Entwicklungsweg Kampucheas wird man keinem Land der Dritten Welt ernsthaft wünschen wollen ${ }^{16}$. Es spricht vielmehr vieles dafür, daß die Beispiele erfolgreicher dissoziativer Entwicklung historisch unwiederbringlich vergangenen Epochen angehören und keine reale Perspektive für die Gegenwart darstellen.

Es ließe sich vielleicht argumentieren, daß die Ơffnung für multinationale Unternehmen in einem sozialistischen Land weniger gefährlich ist, da die Möglichkeiten, ausländische Aktivitäten den eigenen Planentscheidungen unterzuordnen, statt unkontrollierten Prozessen ausgesetzt zu sein, größer ist. Dafür spricht in der Tat einiges, aber wenn man auf diese Weise die Schädlichkeit von Auslandsinvestitionen je nach der institutionellen Kapazität des Anlagelandes relativiert, hat man im Grunde ein dissoziatives Modell aufgegeben. Weltmarktintegration und multinationale Aktivitäten erscheinen dann nicht mehr grundsätzlich als entwicklungspolitische Sackgasse, sondern als mögliche Entwicklungsbeiträge, wenn bestimmte Rahmenbedingungen gesichert sind, und es wäre zu diskutieren, ob das nur in sozialistischen Staaten oder auch in anderen Systemen mit hochentwickelter administrativer und planerischer Kapazität der Fall sein kann. Die Vertreter des Dissoziationsmodells sind vorsichtig genug, eine derartige Strategie („selektive“ Import-Export-Aktivitäten, „Abkoppelung“ nicht „Abschottung“) nicht völlig ausschließen ${ }^{17}$. Damit erhält das Modell Realitätsbezug, wird aber auch relativiert. Dissoziation bei selektiver Außenverflechtung und die diversen Modelle, die die

\footnotetext{
14 Tetzlaff, Die Forderungen der Entwicklungsländer nach einer „neuen * Weltwirtschaftsordnung, VRU 1976, S. $33 \mathrm{ff}$., 36-41; Senghaas, a. a. O., S. $209 \mathrm{ff}$., vgl. auch Hanisch, in diesem Heft, S. 27.

15 Senghaas/Menzel, Multinationale Konzerne und Dritte Welt, in: Senghaas/Menzel, S. 52.

16 Wenn Senghaas a. a. O., S. 21, Kuba unter die Beispiele nautozentrierter Entwicklung* aufzählt, so erscheint das angesichts der Tatsache, daß dieses Land ökonomsich vollständig auf die UdSSR angewiesen ist (mit den entsprechenden Folgen für seinen politischen Handlungsspielraum). immerhin zweif elhaft.

17 Senghaas, a. a. O., S. 277 f.
} 
Position der Entwicklungsländer im Rahmen der Weltwirtschaft stärken wollen, sind keine so absoluten Gegensätze mehr, wie der Schlagabtausch der Theoretiker vermuten lassen könnte. Vor diesem Hintergrund scheint es unangemessen, die Bemühungen um eine "Neue Weltwirtschaftsordnung" als „symbolic use of politics" 18 und als "Der Weltwirtschaftsordnung neue Kleider" abzutun" ${ }^{19}$. Angesichts der Tatsache, daß eine konsequent dissoziative Entwicklungspolitik keine realistische Möglichkeit ist, erscheint es vielmehr nötig, sich auf die „reformistischen" Diskussionen um die Verbesserung der Weltwirtschaftsordnung einzulassen, statt sich dieser Diskussion zu entziehen, indem man ihr die Utopie einer Abkoppelungsstrategie entgegensetzt.

18 Senghaas/Menzel, a. a. O., S. 63.

19 Senghaas, a. a. O., S. 205 ff. 


\section{Réglémentation des \\ INVESTISSEMENTS ETRANGERS EN REPUBLIQUE SOCIALISTE DU VIET NAM*}

\section{Chapitre I \\ DISPOSITION GENERALES}

Article 1. Le Gouvernement de la République Socialiste du Viet Nam accueille favorablement les investissements étrangers sur la base des principes du respect de l'indépendance et de la souveraineté nationale et des avantages réciproques.

Article 2. Est considéré comme investissement étranger au Viet Nam le fait d'introduire et d'utiliser, pour construire de nouvelles entreprises, moderniser les équipements techniques ou élargir les entreprises existantes, les biens et fonds qui suivent:

- tous genres d'équipements, de machines, d'outillage (y compris instruments de laboratoire), de matériel de transport, de matériel technique, etc., indispensables à la réalisation des objectifs susmentionnés.

- tous droits de propriété industrielle: brevets d'invention, procédés technologiques, marques de fabrique, etc ...

- tous capitaux en devises étrangères ou en marchandises ayant valeur de devises, si la Partie Vietnamienne le juge nécessaire.

- tous capitaux en devises étrangères pour rénumérer le personnel et les ouvriers travaillant dans les entreprises ou effectuant des services conformément au présent Code.

Article 3. Les investisseurs étrangers - ci-dessous dénommés la Partie Etrangère - peuvent être des entreprises, des sociétés, des organismes privées ou étatiques, des organisations internationales ou des particuliers remplissant les conditions fixées par le présent Code.

Article 4. La Partie Etrangère peut investir dans le domaine de l'exploitation des ressources, dans l'agriculture, l'industrie, les constructions, les transports . . . à l'exclusion des domaines, des secteurs réservés par le Gouvernement de la République Socialiste du Viet Nam.

\section{Chapitre II \\ FORMES DES INVESTISSEMENTS}

Article 5. La Partie Etrangère peut investir au Viet Nam sous les formes suivantes: 1. Coopération dans la production avec partage des produits;

2. Entreprises ou sociétés mixtes;

3. Entreprises privées spécialisées dans la production des marchandises exclusivement destinées à l'exportation.

Article 6. La coopération dans la production avec partage des produits entre la Partie Vietnamienne et la Partie Etrangère sera réalisée selon les conditions suivantes:

\footnotetext{
* Text der Vietnamesischen Botschaft in Frankreich.
} 
1. La Partie Etrangère apporte les fonds et équipements techniques pour construire une ou plusieurs unités de production, en vue de réaliser un programme de çoopération économique, arrêté d'accord parties.

2. Les équipements, le matériel technique, les droits de propriété industrielle et les autres fonds introduits au Viet Nam par la Partie Etrangère doivent être évalués en espèces par les Parties pour déterminer les apports en capitaux de la Partie Etrangère.

3. Les biens produits sont partagés entre la Partie Vietnamienne et la Partie Etrangère selon un pourcentage et pour une durée fixés d'accord parties. La part réservée à la Partie Etrangère ne peut être vendue au Viet Nam, excepté le cas où la Partie Vietnamienne désire en garder une partie ou la totalité pour la consommation intérieure.

Article 7. L'entreprise ou société mixte sera réalisée selon les conditions suivantes: 1. La Partie Etrangère et une organisation économique étatique vietnamienne constituent un fonds commun pour fonder une entreprise ou société mixte sous forme de société anonyme ou société à responsabilité limitée.

2. L'entreprise ou société mixte est une personne juridique constituée conformément à la législation vietnamienne et fonctionnant selon les dispositions du contrat de société et les status de l'entreprise ou société mixte.

3. L'apport de la Partie Etrangère dans l'entreprise ou société mixte comprend les équipements, les machines, l'outillage, le matériel de transport, le matériel technique, les droits de propriété industrielle et les capitaux en devises étrangères mentionnés à l'Article 2. Cet apport doit constituer au moins 30\% et ne peut dépasser $49 \%$ du capital de la Société.

L'apport de la Partie Vietnamienne comprend notamment les terrains, les installations, les machines, l'outillage, les matériaux de construction et les dépenses effectuées en monnaie vietnamienne.

4. Les apports des Parties doivent être évalués en espèces, dans une monnaie étrangère convenue, et mentionnées dans le contrat de société.

5. Les fonds en espèces de l'entreprise ou socièté mixte doivent être déposés en compte à la Banque du Commerce Extérieur du Viet Nam.

6. Les biens produits par l'entreprise ou société mixte doivent être exportés selon un pourcentage convenue entre les Parties. Les Parties sont également tenues d'effectuer cette exportation dans les conditions les meilleures.

7. Lest status de l'entreprise ou société mixte doivent être enregistrés au Ministère du Commerce Extérieur et au Ministère des Finances de la République Socialiste du Viet Nam.

Les status doivent comprendre les points essentiels suivants:

- Noms des Parties participant à l'entreprise ou société mixte.

- Dénomination de l'entreprise ou société mixte; Siège social.

- Objet de l'entreprise ou société mixte.

- Programme et plan de production et d'activité commerciale; Perspectives de développement.

- Capital; Mode d'apport; Compte en banque.

- Composition et fonctionnement des organes de gestion; Règles relatives à la nomination, l'adjonction et le remplacement des personnes tenant des posteclés de la gestion; Modalités de gestion. 
- Bénéfices; Règles pour la répartition et l'utilisation des bénéfices; Fonds de réserve.

- Fonds d'assurances sociales.

- Cession; Dissolution; Liquidation.

Article 8. Les entreprises privées spécialisées dans la production des marchandises exclusivement destinées à l'exportation doivent se conformer aux conditions suivantes:

1. La Partie Etrangère apporte au Viet Nam les équipements techniques, matières premières, carburants, matériaux de construction, etc. ... nécessaires à la construction et au fonctionnement de l'entreprise, à l'exception de ce que la Partie Vietnamienne est en mesure de fournir, sous forme de vente - achat.

2. L'activité de l'entreprise ne doit en aucune manière porter préjudice à l'économie du Viet Nam.

3. L'exportation des marchandises produites par l'entreprise est soumise au contrôle de la Douane vietnamienne; les devises étrangères provenant de l'exportation doivent être déposées en compte à la Banque du Commerce Extérieur du Viet Nam.

4. Le personnel et les ouvriers de l'entreprise doivent être de nationalité vietnamienne, excepté les cas prévus à l'article 12 du présent Code.

Les salaires du personnel et des ouvriers vietnamiens doivent être payés en devises étrangères.

5. L'entreprise est une personne juridique constituée selon la législation vietnamienne; elle doit faire enregistrer ses statuts au Ministère du Commerce Extérieur et au Ministère des Finances de la République Socialiste du Viet Nam.

\section{Chapitre III}

\section{DROITS ET OBLIGATIONS DE LA PARTIE ETRANGERE}

Article 9. La Partie Etrangère participant à la coopération dans la production avec partage de produits a droit au dit partage conformément aux stipulations du contrat signé avec la Partie Vietnamienne. Cette part de produits est exonérée de taxes à l'exportation.

Article 10. Dans les cas de participation aux entreprises ou sociétés mixtes et d'investissement dans les entreprises privées spécialisées dans la production des marchandises exclusivement destinées à l'exportation, la Partie Etrangère bénéficie des droits suivants:

1. Garantie par le Gouvernement Vietnamien d'une durée d'activité de 10 à 15 ans, à partir du jour où le permis d'investissement est accordé. Cette durée peut être plus longue dans les cas exceptionnels.

2. Protection par le Gouvernement de la République Socialiste du Viet Nam du capital investi. Si en raison des exigences de l'economie nationale, l'entreprise doit être nationalisée, le Gouvernement vietnamien la rachètera selon un prix raisonnable, convenu entre les Parties. Le paiement se fera dans la monnaie investie, dans un délai raisonnable.

3. Cession du capital investi, en totalité ou en partie, après autorisation du Ministère du Commerce Extérieur et du Ministère des Finances de la République conialiste du Viet Nam. 
4. Rapatriement ou transfert à l'étranger:

- des bénéfices nets annuels, déduction faite des impôts et des prélèvements pour constituer le fonds de réserve, jusqu'à concurrence de $25 \%$ du capital investi, lesdits prélèvements sont fixés à raison de $5 \%$ des bénéfices annuels de l'entreprise.

- des fonds récupérés en cas de cessation, de dissolution de l'entreprise ou de rachat par le Gouvernement de la République Socialiste du Viet Nam.

5. Le Gouvernement de la République Socialiste du Viet Nam encourage le réinvestissement des bénéfices au Viet Nam.

Les capitaux réinvestis bénéficient de l'exemption ou de la réduction de l'impôt sur le revenu, selon l'importance des investissements et selon le secteur réinvesti.

Article 11. En dehors des droits prévus à l'article 10, la Partie Etrangère qui investit dans les entreprises ou société mixtes bénéficie des droits suivants:

1. Exonération totale ou réduction de l'impôt sur le revenu pendant des premières années d'exploitation, selon la branche économique, le lieu d'exploitation et l'importance des fonds investis. Cette exonération ou réduction sera décidée pour chaque cas par le Ministère des Finances de la République Socialiste du Viet Nam et mentionnée dans le permis d'investissement.

2. Exonération totale ou réduction éventuelles, pour une ou plusieurs fois, des taxes à l'importation sur les équipements, machines, outillages, pièces de rechange, etc., importés pour équiper les entreprises mixtes, ainsi que les matières premières, carburants, matériaux de construction, etc., nécessaires au fonctionnement de l'entreprise.

3. Exonération totale ou réduction éventuelles des taxes à l'exportation sur les produits de l'entreprise destinés à l'exportation.

4. Au cas où l'entreprise subit un risque imprévisible et insurmontable, la Partie Etrangére peut demander une réduction de l'impôt sur le revenu.

Article 12. En dehors des droits prévus à l'article 10, la Partie Etrangère qui investit dans les entreprises privées spécialisées dans la production des marchandises exclusivement destinées à l'exportation bénéficie des droits suivants:

1. Emploi de la main d'œuvre et des techniciens vietnamiens, conformément à la législation du travail de l'Etat vietnamien et au contrat collectif signé avec les représentants des travailleurs.

2. Emploi des techniciens étrangers, après approbation du Ministère du Commerce Extérieur de la République Socialiste du Viet Nam, dans les travaux et fonctions pour lesquels la Partie Vietnamienne n'est pas offrante.

3. Jouissance de régime d'admission temporaire exemptant de taxes douaniéres les machines, équipements, outillage, pièces de rechange, matières premières, carburants, matériaux de construction, etc., nécessaires au fonctionnement et à l'activité commerciale de l'entreprise.

4. Exemption des taxes à l'exportation des produits de l'entreprise destinés à l'exportation.

5. Relation directe avec les entreprises ou sociétés étrangères, en conformité avec la règlementation du commerce extérieur et du contrôle des changes en vigueur au Viet Nam.

Article 13. La protection des droits de propriété industrielle mentionnés dans l'article 2 présent figurera dans le contrat. 
Article 14. La Partie Etrangère qui investit au Viet Nam sous n'importe quelle forme précitée, est tenue aux obligations suivantes:

1. Respect des lois et règlements en vigueur au Viet Nam et soumission au contrôle des organismes compétents de l'Etat Vietnamien.

2. Application du système de comptabilité vietnamien ou d'un système de comptabilité agréé par le Ministère des Finances de la République Socialiste du Viet Nam. 3. Soumission aux règlementations du commerce extérieur et au contrôle des changes en vigueur au Viet Nam.

4. Exécution complète des clauses du contrat d'investissement.

Article 15. La Partie Etrangère qui investit dans les entreprises ou sociétés mixtes et dans les entreprises privées spécialisées dans la production des marchandises exclusivement destinées à l'exportation est tenue, en plus, aux obligations suivantes: 1. Strict respect de la règlementation sur l'enregistrement des sociétés, des statuts de l'entreprise ou société mixte.

2. Paiement des impôts sur le revenu, fixé à:

- 30\% des bénéfices imposables pour les branches de production exclusivement destinées à l'exportation, prélevés forfaitairement sur le chiffre d'affaires ou sur les unités de biens produits.

- $40 \%$ des bénéfices imposables pour les branches économiques nécessitant un niveau technique avancé et des investissements importants.

- $50 \%$ des bénéfices imposables pour les autres branches économiques.

Article 16. Toute fraude ou tout acte déloyal commis par la Partie Etrangère au cours de l'exécution du contrat d'investissement est sanctionné, selon la gravité de la faute, par des amendes ou par le retrait de certains droits stipulés aux articles 10 , 11 et 12 du présent Code.

En cas d'infractions graves, telles que l'interruption injustifiée de l'exécution du contrat, le permis d'investissement peut être retiré sans dédommagement.

\section{Chapitre IV}

\section{PROCEDURE}

Article 17. Les demandes d'investissement au Viet Nam doivent être adressées au Ministère du Commerce Extérieur de la République Socialiste du Viet Nam accompagnées des documents nécessaires et du paiement des frais règlementaires fixés par ledit Ministère.

Article 18. Les demandes sont examinées et font l'objet d'une décision pour chaque cas séparément, dans un délai de trois mois, à partir de la date à laquelle le Ministère du Commerce Extérieur de la République Socialiste du Viet Nam accuse réception de la demande et du dossier en règle.

Article 19. Le permis d'investissement sera délivré par le Ministère du Commerce Extérieur de la République Socialiste du Viet Nam.

Article 20. Munie de permis, la Partie Extrangère doit faire enregistrer l'acte d'investissement au Ministère du Commerce Extérieur et au Ministère des Finances de la République Socialiste du Viet Nam. 


\title{
Chapitre V \\ DISSOLUTION ET LIQUIDATION DES ENTREPRISES ET SOCIETES AYANT DES INVESTISSEMENTS ETRANGERES
}

Article 21. Toute entreprise, société ayant des investissements étrangers dont la dissolution est survenue par suite de l'expiration du contrat d'investissement ou pour toute autre cause, doit être liquidée conformément à la législation vietnamienne, au contrat d'investissement et aux statuts de ladite entreprise.

Les droits du personnel vietnamien travaillant dans l'entreprise ou dans la société doivent être assurés conformément à la législation vietnamienne.

Article 22. Au cas où la dissolution de l'entreprise ou de la société est dûe à une infraction grave ou à une violation du contrat d'investissement par la Partie Etrangère, celle-ci supportera tous les frais et charges occasionnés par cette dissolution.

Article 23. La Partie Etrangère doit faire enregistrer la cessation de l'investissement au Ministère du Commerce Extérieur et au Ministère des Finances de la République Socialiste du Viet Nam.

\section{Chapitre VI}

\section{REGLEMENT DES LITIGES}

Article 24. Les litiges entre les entreprises, sociétés ayant des investissement étrangers d'une part, et les organismes économiques vietnamiens ou le personnel vietnamien travaillant dans lesdites entreprises, société ou tout autre citoyen vietnamien d'autre part, seront tranchés soit par la Commission d'arbitrage économique, soit par les services compétents du Travail, soit par les tribunaux compétents selon la nature du litige et conformément à la législation vietnamienne.

Article 25. Les litiges issus des rapports contractuels entre la Partie Etrangère et la Partie Vietnamienne seront tranchés par la Commission d'arbitrage du Commerce Extérieur près la Chambre de Commerce du Viet Nam, excepté les cas particuliers où les Parties auront convenu d'un autre mode d'arbitrage dûment mentionné dans le contrat d'investissement.

\section{Chapitre VII \\ DISPOSITIONS D’APPLICATIONS}

Article 26. Dans les cas particuliers et si nécessaire, le Gouvernement de la République Socialiste du Viet Nam pourra accorder à la Partie Etrangère des conditions plus favorables que celles mentionnées précédemment.

Article 27. Le Ministère du Commerce Extérieur, le Ministère des Finances, le Ministère du Travail, la Banque d'Etat du Viet Nam sont chargés de fixer les dispositions concrètes en vue de l'application du présent Code.

\author{
POUR LE CONSEIL DU GOUVERNEMENT \\ DE LA REPUBLIQUE SOCIALISTE DU VIET NAM \\ Le Premier Ministre
}

